\title{
Integrated Transcriptomic and Metabolomic analysis reveals a transcriptional regulation network for the biosynthesis of carotenoids and flavonoids in 'Cara cara' navel Orange
}

Haipeng Zhang, Jiajing Chen, Zhaoxin Peng, Meiyan Shi, Xiao Liu, Huan Wen, Youwu Jiang, Yunjiang Cheng, Juan $\mathrm{Xu}^{*}$ and Hongyan Zhang ${ }^{*}$

\begin{abstract}
Background: Carotenoids and flavonoids are important secondary metabolites in plants, which exert multiple bioactivities and benefits to human health. Although the genes that encode carotenogenesis and flavonoid biosynthetic enzymes are well characterized, the transcriptional regulatory mechanisms that are related to the pathway genes remain to be investigated. In this study, 'Cara cara' navel orange (CNO) fruit at four development stages were used to identify the key genes and TFs for carotenoids and flavonoids accumulation.

Results: In this study, CNO was used to investigate the profiles of carotenoids and flavonoids by a combination of metabolomic and transcriptomic analyses. The important stage for the accumulation of the major carotenoid, lycopene was found to be at 120 days after florescence (DAF). The transcripts of five carotenogenesis genes were highly correlated with lycopene contents, and 16, 40, 48, 24 and 18 transcription factors (TFs) were predicted to potentially bind 1-deoxy-D-xylulose-5-phosphate synthase (DXS1), deoxyxylulose 5-phosphate reductoisomerase (DXR), geranylgeranyl diphosphate synthase (GGPPS2), phytoene synthase (PSY1) and lycopene $\beta$-cyclase (LCYB) promoters, respectively. Narirutin was the most abundant flavonoid in the flesh at the early stages, 60 DAF was the most important stage for the accumulation of flavonoids, and 17, 22, 14, 25, 24 and 16 TFs could potentially bind phenylalanine ammonia-lyase (PAL-1 and PAL-4), 4-Coumarate-CoA ligase (4CL-2 and 4CL-5), chalcone synthase (CHS1) and chalcone isomerase $(\mathrm{CH})$ promoters, respectively. Furthermore, both sets of 15 candidate TFs might regulate at least three key genes and contribute to carotenoids/flavonoids accumulation in CNO fruit. Finally, a hierarchical model for the regulatory network among the pathway genes and TFs was proposed.
\end{abstract}

Conclusions: Collectively, our results suggest that DXS1, DXR, GGPPS2, PSY1 and LCYB genes were the most important genes for carotenoids accumulation, while PAL-1, PAL-4, 4CL-2, 4CL-5, CHS-1 and CHI for flavonoids biosynthesis. A total of 24 TFs were postulated as co-regulators in both pathways directly, which might play important roles in carotenoids and flavonoids accumulation in CNO fruit.

Keywords: Citrus, Carotenoid, Flavonoid, Transcription factor, Transcriptome, Metabolomics

\footnotetext{
*Correspondence: xujuan@mail.hzau.edu.cn; zhanghy@mail.hzau.edu.cn Key Laboratory of Horticultural Plant Biology (Ministry of Education), College of Horticulture and Forestry, Huazhong Agricultural University, Wuhan 430070, People's Republic of China
} 


\section{Background}

Citrus is one of the most important fruits in the world, and is rich in various primary and secondary metabolites, such as amino acids, organic acids, soluble sugars, flavonoids, volatile terpenoids, limonoids, coumarins and carotenoids [1-5]. Some metabolites, including flavonoids, limonoids, organic acids and soluble sugars, were reported to contribute to fruit taste [3, 4], whilst terpenoids and carotenoids were associated with fruit aroma and color $[2,6]$.

Carotenoids not only contribute to the eye-attracting fruit flesh color, but also exhibit various bioactivities in human body, such as prevention of cancer and other diseases [7] and acting as the precursors for vitamin A [8]. In citrus, the flesh color mainly depends on the contents of carotenoids [6], with flavonoids and anthocyanins exerting yellow background and blood orange respectively $[9,10]$. The contents of major carotenoids are varied in different citrus species, $\beta$-cryptoxanthin is the major carotenoid in loose-skin mandarin (Citrus reticulata), violaxanthin is the most abundant in sweet oranges (C. sinensis), while lower levels of carotenoids were found in most of pomelo (C. grandis) and citrons (C. medica) [11]. Moreover, some citrus germplasms are rich in special carotenoids in their specific tissues. For example, 'Red tangerine' peel is red, with abundant $\beta$ citraurin [12]; The flesh of 'Hong Anliu' sweet orange (C. sinensis) and 'Cara cara' navel orange (C. sinensis, $\mathrm{CNO}$ ) is pink or red due to their high concentrations of lycopene [6]. Lycopene is an excellent antioxidant that plays important roles in skin protection, cardiovascular disease prevention, and anti-aging and anti-cancer activities [13]. A high concentration of lycopene in citrus fruit not only improves the fruit quality but is also beneficial to human health.

In higher plants, the isomeric $\mathrm{C} 5$ isopentenyl pyrophosphate (IPP) is synthesized in 2-C-methyl-D-erythritol-4-phosphate (MEP) pathway, and is used as the precursor of geranylgeranyl diphosphate (GGPP) by GGPP synthase gene, GGPPS. GGPP is the direct precursor for various carotenoids catalyzed by phytoene synthase (PSY), phytoene desaturase (PDS), $\zeta$-carotene desaturase (ZDS), lycopene $\beta$-cyclase (LCYB), carotene isomerase (CRTISO), carotene hydroxylase (HYD), violaxanthin de-epoxidase (VDE) and zeaxanthin epoxidese (ZEP), etc. [8]. Previous studies have shown that 1deoxy-D-xylulose-5-phosphate synthase (DXS) was an important rate-limiting enzyme in the MEP pathway in tomato, Arabidopsis and Kiwifruit [14, 15]. Besides, the deoxyxylulose 5-phosphate reductoisomerase (DXR) was shown to be rate-limiting enzyme in Arabidopsis, overexpression of DXR increased the content of carotenoids [16]. PSY is the key rate-limiting enzyme in the biosynthetic pathway of carotenoids in tomato and citrus [17,
18]. The contents of carotenoids were significantly increased when $P S Y$ was over-expressed in both tomato and citrus fruit $[17,18]$. In tomato fruit, DXS, PSY, PDS, $Z D S$ and CRTISO are up-regulated at the mature stage, resulting in lycopene's over-accumulation [19]. The expression of DXS was positively correlated with the content of carotenoids in citrus [20]. 'Hong Anliu' sweet orange is a red flesh mutant from 'Anliu' sweet orange, with its fruit accumulating lycopene around 180 DAF [20]. It was found that some biosynthetic pathway genes and transcription factors (TFs) contributed to the accumulation of lycopene by combining transcriptome, proteomic and metabolomic analyses [17, 20, 21]. However, to our knowledge, the molecular basis of lycopene over-accumulation is still not clear.

Although the genes that encode carotenogenesis enzymes are well characterized, the transcriptional regulatory mechanisms that are related to the pathway genes remain to be investigated. TFs directly regulate the expression levels of their target genes, and take part in various biological processes in plants. Some TFs have been found to be associated with the accumulation of carotenoids, such as TAGL1, FUL1/2 and ERF6 [22-24]. Lycopene level was increased by over-expression of TAGL1 or PpPLENA in tomato fruit [22-24], while decreased in TAGL1 or FUL1/2 loss of function mutant [24]. Some TFs can directly bind to the biosynthetic pathway genes' promoters, then affect the accumulation of carotenoids. For example, PIF1 and SIMADS1 specially bound the PSY promoter and decreased its transcription and thus altered the carotenoids profile [25, 26], whereas $H Y 5$ and RIN up-regulated the expression of $P S Y$ in tomato [27]. In addition, the CrMYB68 decreased the expression levels of $\mathrm{CrBCH} 2$ and $\mathrm{CrNCED} 5$ in citrus [28], whist CsMADS6 increased those of $L C Y b 1$, PSY and PDS in 'Hong Anliu' [17].

Cara cara navel orange $(\mathrm{CNO})$ is a bud mutant with red-flesh derived from Washington navel orange, due to the over-accumulation of lycopene and the high content of $\beta$-carotene. Similar results were found with some red flesh grapefruits and sweet oranges, but with varied ratios of lycopene to $\beta$-carotene [6], indicating the different patterns of carotenoids within different genotypes. It was found that the expression levels of DXS, HDS and $H D R$ in $\mathrm{CNO}$ were higher than those in Washington navel orange at the mature stage [29]. However, the reason for the up-regulation of these genes remains unknown.

Flavonoids, one of the most important secondary metabolites in citrus fruit, have multiple functions, such as anti-cancer, anti-virus, anti-inflammatory and antibacterial activities [30, 31]. Flavonoids mainly include flavanone, flavones, flavonol, dihydroflavonol and anthocyanin, and the flavanone glycosides are the major 
flavonoids in citrus fruit. Our previous study found that the contents of flavonoids in four sweet oranges tended to be stable or slightly decreased during 90-210 DAF, and that the lycopene accumulation may have direct or indirect effects on the biosynthesis of flavonoids [9]. The flavonoids are synthesized from phenylpropanoid pathway, mainly including $P A L, C 4 H, 4 C L, C H S$ and $\mathrm{CHI}$ genes [32]. It was found that the expression levels of these genes were at high levels at 60 DFA, but simultaneously dropped to extremely low levels at 90-210 DAF in four pomelos, the key pathway genes might be regulated by some TFs [32]. Numerous TFs have been reported to regulate lignin and anthocyanin biosynthesis in phenylpropanoid pathway [33]. For example, CsMYBF1 was found to bind to CHS-1 promoter and then affect flavanone accumulation in citrus [34]; Ruby was found to bind to CHS, DFR and ANS promoters, then regulate anthocyanin accumulation in blood orange and Zipi pomelo [10, 35]. Furthermore, many MYB TFs were reported to directly bind and regulate $P A L$ and $C 4 H$ promoter to affect lignin accumulation in Arabidopsis and sweet orange $[36,37]$. However, to our knowledge, the regulatory mechanisms of TFs in the biosynthesis of both flavonoids and carotenoids remain unclear in citrus fruit.

In this study, HPLC-based carotenoids and flavonoids profiling combined with RNA-seq based gene expression data is utilized to mine the important pathway genes and TFs contributing to carotenoids and flavonoids profiles in juice sacs during fruit development of CNO fruit. The results may provide new insights into the mechanism governing specific carotenoids and flavonoids accumulation in citrus fruit.

\section{Results}

Carotenoids and flavonoids profiles in juice sacs of CNO during fruit development

Juice sacs at six different developmental stages of $\mathrm{CNO}$ were determined for variation in carotenoids and flavonoids profiles using HPLC. The contents of both carotenoids and flavonoids varied greatly at different developmental stages.

Seven major carotenoids were identified, including lycopene, phytoene, phytofluene, lutein, violaxanthin, $\beta$ cryptoxanthin and $\beta$-carotene, with the level of lycopene being the highest (Additional file 1: Table S1; Fig. 1a). The content of lycopene was low at $60 \mathrm{DAF}$, and significantly increased from 90 to $120 \mathrm{DAF}$, reached to a maximum at $150 \mathrm{DAF}$, then significantly decreased at the mature stage (Fig. 1a). The increases of lycopene levels were $2.65 \mu \mathrm{g} / \mathrm{g}, 23.23 \mu \mathrm{g} / \mathrm{g}$ and $14.19 \mu \mathrm{g} / \mathrm{g}$ from 60 to 90 , 90 to 120 and 120 to 150 DAF, respectively, indicating that the stage from 90 to 120 DAF might be the most important for the lycopene biosynthesis (Additional file 1: Table S1; Fig. 1a). For the other carotenoids identified, phytoene and phytofluene were also significantly increased from 90 to 120 DAF, then maintained or slightly decreased at the mature stage (Fig. 1a). Lutein was found at 180 DAF and its level increased at 210 DAF; $\beta$ cryptoxanthin and $\beta$-carotene were detected at $150 \mathrm{DAF}$ and tended to increase from 150 to 210 DAF; violaxanthin was detected at $120 \mathrm{DAF}$, then significantly increased from 150 to 180 DAF (Additional file 1: Table S1).

A total of 11 flavonoids were identified in CNO fruit at four development stages, with narirutin as the dominant one at 60 and 90 DAF (Additional file 1: Table S2).
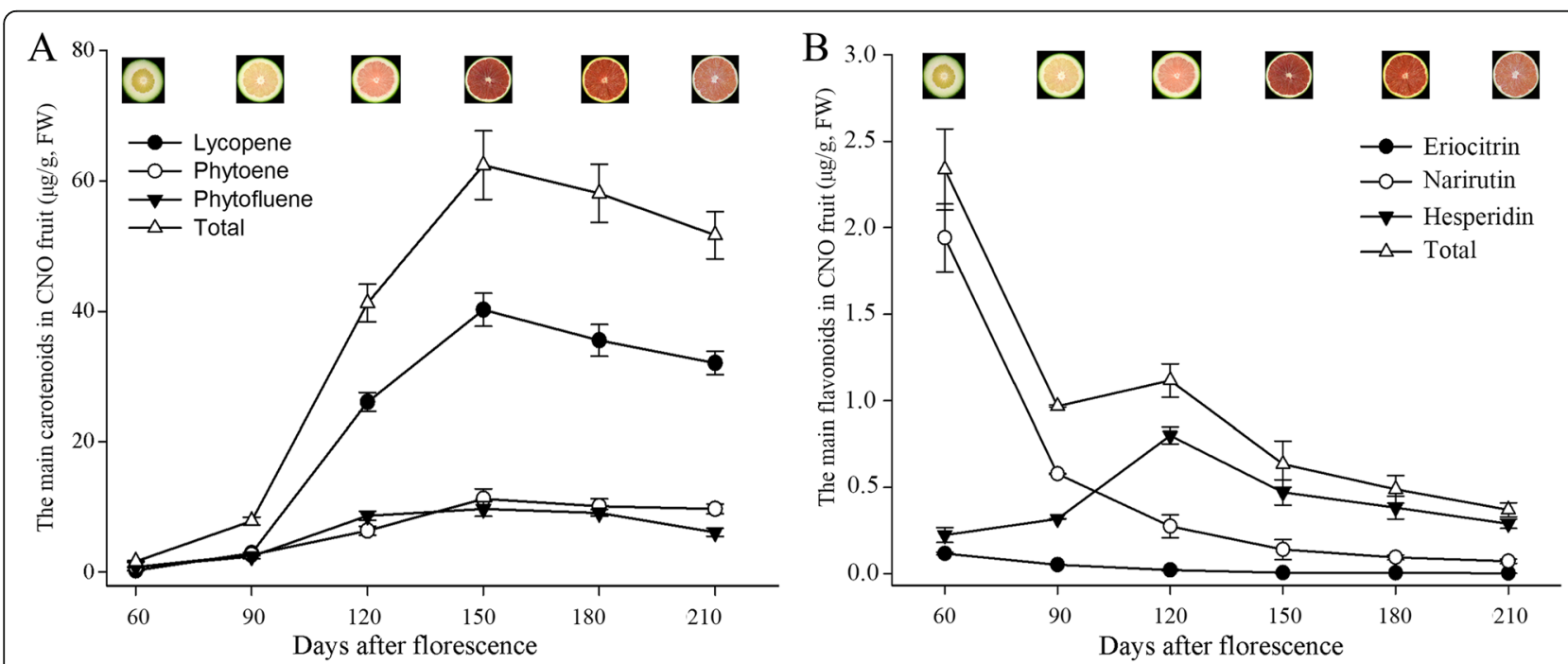

Fig. 1 The main carotenoids and flavonoids identified in CNO juice sacs during fruit development. a, The main carotenoids in CNO fruit ( $\mu$ g/g, FW); b. The main flavonoids in CNO fruit $(\mu \mathrm{g} / \mathrm{g}, \mathrm{FW})$ 
The contents of total flavonoids were the highest and lowest at $60 \mathrm{DAF}$ and $210 \mathrm{DAF}$, respectively. The results suggested that the 60 DAF stage might be the important stage for the accumulation of flavonoids (Fig. 1b).

Pearson correlation coefficient was -0.797 between total carotenoids and total flavonoids levels in $\mathrm{CNO}$ fruit at six development stages, while -0.719 was at 60 to 150 DAF, suggesting a negative correlation between total contents of carotenoids and flavonoids. Furthermore, before $150 \mathrm{DAF}$, the level of carotenoids constantly increased, whereas the content of flavonoids sharply decreased at 90 DAF with a slight increase at 120 DAF due to the increase of hesperidin content. After 150 DAF, the level of lycopene significantly decreased, whilst the levels of the other main carotenoids and all flavonoids decreased or kept at stable levels (Fig. 1). These results showed that there were collaborative changes in carotenoids and flavonoids biosynthesis, with 150 DAF as the turning stage.

\section{Differentially expressed genes in CNO during fruit development}

To further determine the expression levels of the genes related to lycopene over-accumulation in $\mathrm{CNO}$, the juice sacs at four developmental stages $(60,90,120$ and 150 DAF) were subjected to transcriptomic analysis. Total of 282.14 M high-quality base pairs were obtained, with an average of $23.51 \mathrm{M}$ data per sample. Approximately 94.68-97.57\% reads were mapped to the Valencia sweet orange genome, among them, $87.22-93.13 \%$ was uniquely aligned, and $4.44-7.46 \%$ was aligned to multiple loci (Additional file 1: Table S3). Based on their expression levels, the average fragments per kilobase of transcript per million fragments mapped (FPKM) higher than 0.5 were selected for further analysis (Additional file 1: Table S4). Totally there were 17,190 genes expressed in juice sacs at four development stages of CNO, with 16,454, 16,170, 15,937 and 15,911 expressed at 60, 90, 120 and 150 DAF, respectively (Fig. 2a), while $178,32,20$ and 187 genes were specially expressed at 60 , 90, 120 and 150 DAF, respectively (Fig. 2b). Furthermore, $88.61,84.62,85.18$ and $87.93 \%$ of expressed genes ranged from 1 to 100 FPKM in 60, 90, 120 and 150 DAF, respectively (Fig. 2a). Principal component analysis (PCA) and correlation analysis showed good repeatability among biological replicates (Fig. 2 c, d).

A total of 2482, 4059, 5565, 3738, 5671 and 3555 differentially expressed genes (DEGs) at 60 DAF vs 90 DAF, 60 DAF vs 120 DAF, 60 DAF vs 150 DAF, 90 DAF vs 120 DAF, 90 DAF vs 150 DAF and 120 DAF vs 150 DAF with the fold change $(|\log 2 \mathrm{FC}| \geq 1)$ and FDR $\leq$ 0.05 were obtained, respectively. For instance, compared with the fruit at $90 \mathrm{DAF}, 2063 / 1675$ genes were significantly up-regulated/down-regulated in the fruit at 120 DAF (Additional file 3: Fig. S1 A).

\section{Important pathway genes for lycopene accumulation}

The stage from 90 to 120 DAF was the most important stage for lycopene accumulation based on metabolic data. Thus, the DEGs were subjected to KEGG enrichment analysis, and the DEGs were found to be mainly enriched in metabolic pathways, biosynthesis of secondary metabolites and plant hormone signal transduction (Additional file 3: Fig. S1 B). Among the DEGs, some genes were clustered into carotenoids biosynthetic and terpenoid backbone biosynthesis, including DXS1 (Cs1g20530), DXS2 (Cs9g05150), HDS (Cs8g16700), HDR (Cs8g07020) and ZDS4 (Cs3g11060), and their expression levels in the fruit at 120 DAF were significantly higher than thoseat90 DAF. Furthermore, KEGG enrichment analysis on the DEGs at 90-120 DAF found that some genes were clustered into plant hormone signal transduction, such as Cs9g08850 (ethylene signal transduction), Cs1g17210 (jasmonate signal transduction) and Cs9g18020 (abscisic acid signal transduction), etc.: which might also play important roles in lycopene overaccumulation (Additional file 3: Fig. S1 B).

To mine the most important genes for the overaccumulation of lycopene during fruit development, all the expressed genes were grouped using Mfuzz analysis based on their expression pattern changes, which yielded nine clusters (Fig. 3; Additional file 1: Table S5). Notably, Cluster 2, 4 and 7 showed a good positive correlation with the content of lycopene, and 1804, 1604 and 1546 genes were grouped in Cluster 2, 4 and 7, respectively (Fig. 3). Further gene function annotation found that DXS1, PSY1 and ZDS2 (Cs3g11060) were in Cluster4, DXR and GGPPS2 were in Cluster2, and $M D S$ (Cs5g03050) was in Cluster7, implying their importance as candidate genes accounting for lycopene overaccumulation. In addition, $L C Y B$ (orange1.1 t00772) with consistently low expression levels was in Cluster 6 (Fig. $3)$.

Next, correlation analysis between the genes within carotenoid biosynthetic pathway and the content of lycopene showed that DXS1, DXR, GGPPS2 and PSY1 were highly and positively related to lycopene levels, with correlation coefficients of $0.997,0.893,0.834$ and 0.964 , respectively, while the values of the other lycopene biosynthesis related genes were lower than 0.800 (Table 1; Fig. 4). Herein, DXS1, DXR, GGPPS2 and PSY1 might be the key biosynthetic pathway genes accounting for the high level of lycopene in juice sacs of $\mathrm{CNO}$ fruits.

\section{TFs related to key carotenoid biosynthetic genes}

To better understand the potential role of TFs in the regulation of DXS1, DXR, GGPPS2, LCYB and PSY1, and 

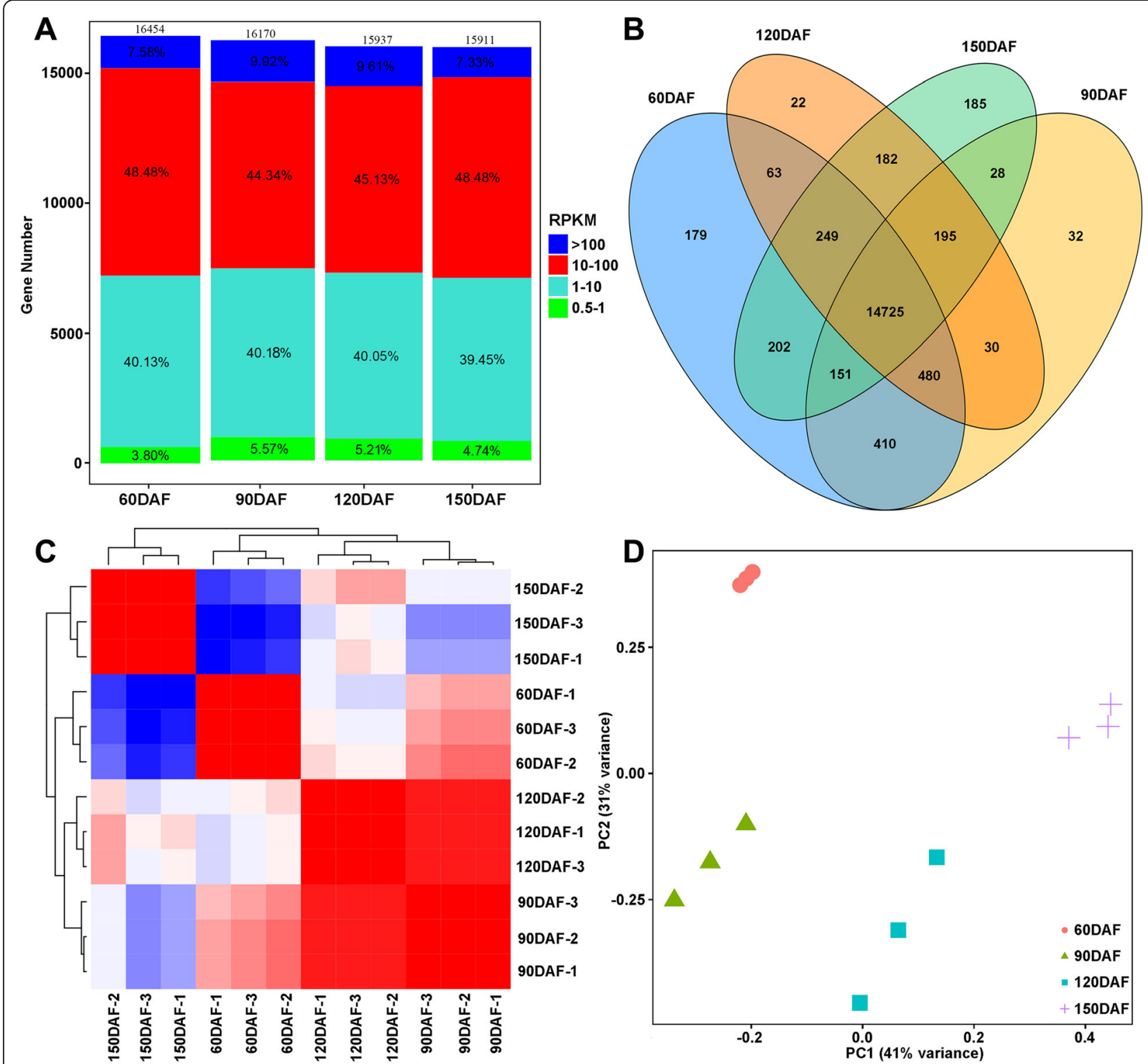

Fig. 2 Basic information obtained from transcriptomic data. a, Expression levels of genes based on RNA-Seq data from juice sacs of CNO at 4 different development stages. b. The specially expressed genes in juice sacs of CNO at 4 different development stages. c: Correlation analysis matrix among CNO at 4 different development stages based on RNA-Seq data. d: PCA analysis on CNO at 4 different development stages based on RNA-Seq data

thus contributing to lycopene accumulation, their promoters were used to predict the possible binding sites using PlantRegMap (Plant TF Database). Potentially 83, 147, 183, 104 and 228 TF binding site in DXS1, DXR, GGPPS2, LCYB and PSY1 promoters were predicted, respectively (Additional file 2: Table S6). However, 16, 40, 48, 18 and 24. TFs had high Pearson correlation coefficients $(|\mathrm{r}|>0.85)$, with 63 and 83 as potential positive and negative regulators respectively (Fig. 5; Additional file 2: Table S7). Among the 146 potential regulators, there were 93 TFs (17 TFs from AP2 family, 12 from MADS, 11 from GRAS, eight from MYB, five from TCP,
WRKY and bZIP, four from SBP, three from ERF, HDZIP and NAC) (Additional file 2: Table S7).

Some TFs were highly positively or negatively correlated with DXS1, DXR, GGPPS2, LCYB and PSY1 genes that contribute to carotenoids accumulation. These TFs might regulate the expression levels of five important genes that contribute to lycopene accumulation in $\mathrm{CNO}$ fruit. For example, DXS1 was positively correlated with $10 \mathrm{TFs}$, and negatively correlated with six TFs, and the coefficients of seven TFs were higher than 0.900 , while four were lower than -0.900 (Fig. 5; Additional file 2: Table S7). 

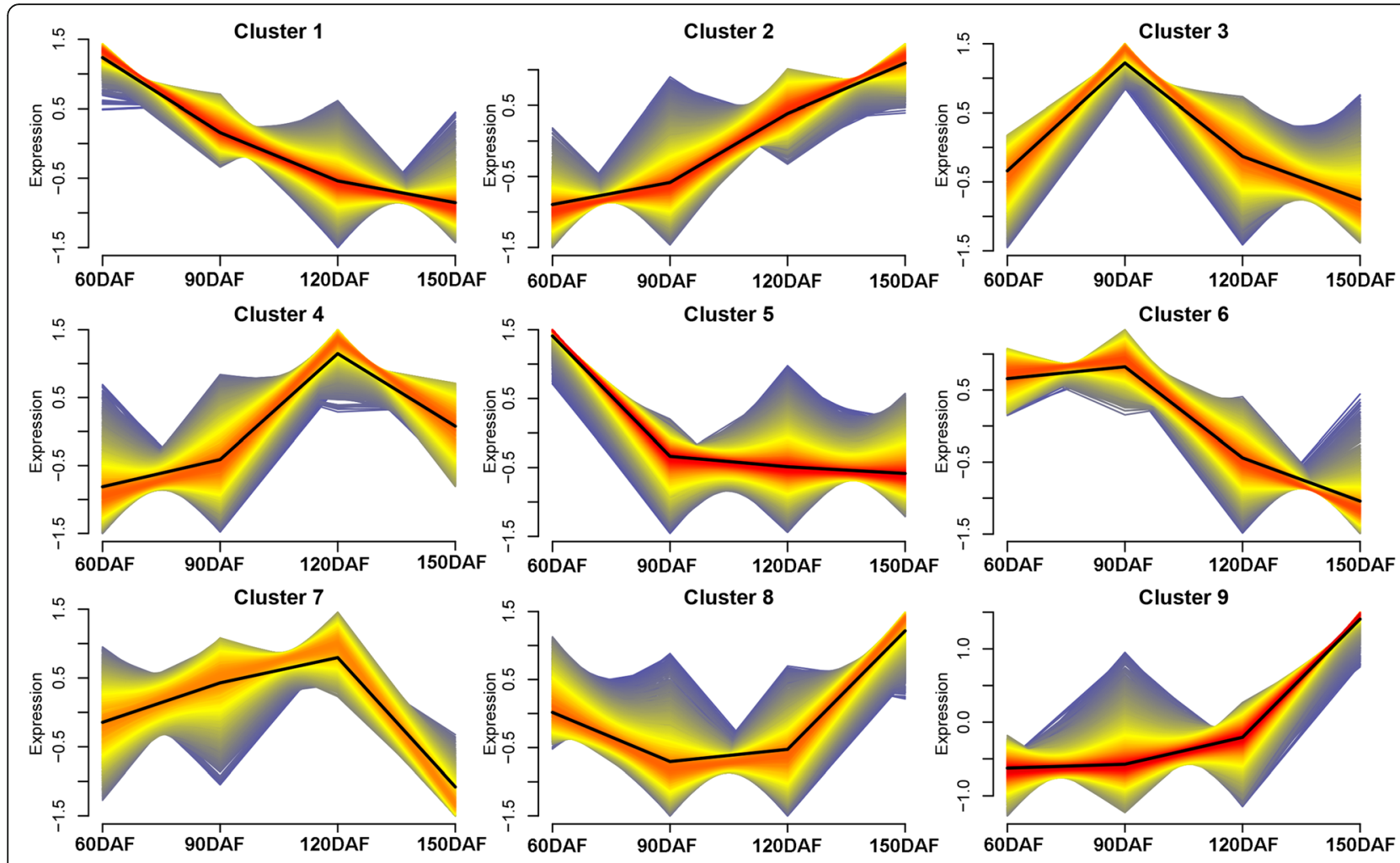

Fig. 3 Expressed genes were clustered into nine expression patterns using Mfuzz in CNO at four developmental stages

Some potential TFs bind to multiple genes, with high Pearson correlation coefficients. For example, Cs3g19420, Cs3g23270, Cs5g26720, Cs7g11810 and Cs7g26660 might positively regulate the four important pathway genes, while Cs9g16575 might be a negative regulator. Some TFs could regulate three of five pathway genes, such as Cs5g23210, Cs5g33540, Cs9g03820 and orange1.1 t02314 which might be potential positive regulators, while Cs4g09270, Cs6g17530, orange1.1 t00456, orange1.1 t02436 and Cs9g07440 might be negative ones (Fig. 5; Additional file 2: Table S7).

\section{Important pathway genes and TFs for flavonoids biosynthesis}

The contents of flavonoids tended to decrease during fruit development (Fig. $1 \mathrm{~B}$ ). The expression levels of the most flavonoids biosynthetic genes were found to be high at 60 DAF in CNO, while dropped at 120 and 150 DAF, including PAL-1, PAL-3, 4CL-2, 4CL-5, CHS-1 and $\mathrm{CHI}$ (Additional file 4: Fig. S2). Results of Mfuzz analysis of Cluster1 showed that their expression tended to decrease (Fig. 3), and KEGG annotation found that the genes in the cluster were mainly involved in metabolic pathways, biosynthesis of secondary metabolites and phenylpropanoid biosynthesis (Additional file 5: Fig. S3). Coincidently, PAL-1, PAL-3, 4CL-2, 4CL-5, CHS-1 and
CHI grouped into Cluster1 were identified via gene annotation.

To further identify the potential TFs that may regulate the above six pathway genes and the accumulation of flavonoids, TFs were predicted using PlantRegMap. The results showed that there were potentially 213, 229, 95, 262, 227 and $102 \mathrm{TF}$ binding sites in $P A L-1, P A L-3$, $4 C L-2,4 C L-5, C H S-1$ and $C H I$ promoters, respectively (Additional file 2: Table S8). The pathway genes with its potential 17, 22, 14, 25, 24 and 16 TFs were found to have high Pearson correlation coefficients $(|\mathrm{r}|>0.85)$, with 111 and seven as potential positive and negative regulators, respectively (Fig. 6; Additional file 2: Table S9). Among the 118 potential regulators, there were 64 TFs, mainly including nine TFs from WRKY family, eight from MYB, six from bHLH and four from ERF (Additional file 2: Table S9).

Some TFs were highly positively or negatively correlated with $P A L-1, P A L-3,4 C L-2,4 C L-5, C H S-1$ and $C H I$ genes that contribute to flavonoids accumulation. These TFs might regulate the expression levels of the six important pathway genes that contribute to flavonoids accumulation in CNO fruit. For instance, PAL-1 was highly and positively correlated with 16 TFs, while negatively correlated with one TF, among them, the Pearson correlation coefficients of 12 TFs were higher than 0.900 (Fig. 6; Additional file 2: Table S9). 
Table 1 Pearson correlation coefficients between the increases of lycopene levels and carotenoid biosynthetic genes

\begin{tabular}{|c|c|c|c|c|c|c|}
\hline GenelD & GeneSymbol & CNO60 & CNO90 & CNO120 & CNO150 & $\mathrm{PCC}^{\mathrm{a}}$ \\
\hline Cs1g20530 & DXS1 & 10.89 & 20.19 & 78.62 & 45.66 & 0.997 \\
\hline Cs9g05150 & DXS2 & 7.11 & 1.93 & 6.16 & 10.08 & 0.343 \\
\hline Cs5g05440 & DXR & 35.04 & 35.42 & 61.87 & 64.87 & 0.893 \\
\hline Cs3g01420 & MCT & 29.61 & 30.12 & 25.74 & 16.03 & -0.541 \\
\hline Cs2g28970 & CMS & 40.14 & 26.31 & 21.28 & 33.00 & -0.631 \\
\hline Cs5g03050 & HDS & 125.48 & 142.94 & 168.68 & 97.26 & 0.352 \\
\hline Cs8g16700 & MDS & 33.72 & 37.99 & 104.16 & 158.33 & 0.743 \\
\hline Cs5g28200 & HDR1 & 48.45 & 50.28 & 52.16 & 48.77 & 0.793 \\
\hline Cs8g07020 & HDR2 & 0.18 & 0.45 & 1.51 & 4.87 & 0.511 \\
\hline Cs6g02690 & $|D|$ & 120.99 & 124.26 & 133.57 & 177.28 & 0.448 \\
\hline Cs1g23670 & GGPPS1 & 103.41 & 77.77 & 63.99 & 51.92 & -0.738 \\
\hline Cs8g02140 & GGPPS2 & 16.90 & 10.97 & 26.47 & 27.88 & 0.834 \\
\hline Cs6g15910 & PSY1 & 44.65 & 58.84 & 89.89 & 66.70 & 0.964 \\
\hline orange1.1 t02108 & PSY2 & 0.53 & 1.34 & 1.53 & 1.70 & 0.784 \\
\hline Cs5g20250 & PDS & 3.45 & 5.41 & 3.99 & 3.42 & -0.250 \\
\hline Cs3g11180 & ZDS1 & 9.86 & 3.00 & 8.33 & 37.89 & 0.408 \\
\hline Cs3g11060 & ZDS2 & 3.15 & 0.00 & 1.27 & 1.87 & -0.280 \\
\hline orange1.1 t05048 & ZDS3 & 2.76 & 3.21 & 6.22 & 1.64 & -0.159 \\
\hline Cs3g11090 & ZDS4 & 3.01 & 1.17 & 3.00 & 1.92 & 0.321 \\
\hline Cs6g13340 & CRTISO & 6.93 & 5.62 & 6.17 & 12.52 & 0.163 \\
\hline Cs4g 14850 & LCYE & 2.48 & 2.66 & 5.38 & 4.94 & 0.943 \\
\hline orange 1.1 t00772 & LCYB & 8.39 & 10.57 & 7.73 & 4.66 & -0.476 \\
\hline Cs9g19270 & HYD & 141.38 & 275.22 & 632.9 & 847.80 & 0.805 \\
\hline Cs1g22620 & ZEP1 & 5.68 & 3.13 & 23.67 & 19.12 & 0.969 \\
\hline orange 1.1 t04050 & ZEP2 & 4.29 & 1.14 & 1.62 & 1.58 & -0.546 \\
\hline Cs2g03270 & NCED1 & 20.98 & 61.70 & 42.32 & 78.31 & 0.322 \\
\hline Cs5g14370 & NCED2 & 26.11 & 6.98 & 2.50 & 5.49 & -0.711 \\
\hline Cs7g01700 & NCED3 & 3.71 & 1.11 & 1.02 & 0.65 & -0.744 \\
\hline Cs7g01710 & NCED4 & 92.84 & 117.49 & 152.99 & 148.52 & 0.933 \\
\hline Cs7g14820 & NCED5 & 6.33 & 13.39 & 5.24 & 11.45 & -0.338 \\
\hline Cs8g14150 & NCED6 & 3.52 & 0.89 & 9.75 & 0.67 & 0.661 \\
\hline
\end{tabular}

$P C C^{a}$ : Pearson correlation coefficient

Among the potential TFs that had high Pearson correlation coefficients with the key pathway genes, Cs1g23760 and Cs3g16330 might positively regulate the five of six key pathway genes, while Cs2g16940, Cs7g04700, Cs6g01750 and orange1.1 t01021 might positively regulate the four out of six pathway genes, and nine TFs (Cs1g08440, Cs3g23190, Cs4g06100, Cs5g12070, Cs5g33880, Cs6g16070, Cs7g03980, Cs9g02700 and orange1.1 t00472) could bind three out of six pathway genes as positive regulators (Fig. 6; Additional file 2: Table S9). These TFs might play important roles in flavonoids biosynthesis.

\section{Postulated hierarchical network in regulating carotenoids and flavonoids profiles in CNO}

Both sets of 15 candidate TFs could bind at least three carotenoids and flavonoids biosynthesis genes, respectively.
Further analysis identified 24 TFs that could regulate important genes involved in both carotenoids and flavonoids biosynthesis pathways. Twenty of these TFs might positively and negatively regulate flavonoid biosynthesis and carotenogenesis genes, respectively, whereas two of them had the opposite effects and the other two were positively correlated with the important genes in both biosynthetic pathways (Additional file 6: Fig. S4). Thus, these TFs might directly regulate the genes related to the biosynthesis pathways for both carotenoids and flavonoids.

\section{Discussion}

The key stage for lycopene and carotenoids accumulation Carotenoids were the important primary and secondary metabolites in plants [38]. Seven main carotenoids were detected in CNO juice sacs using HPLC (Additional file 


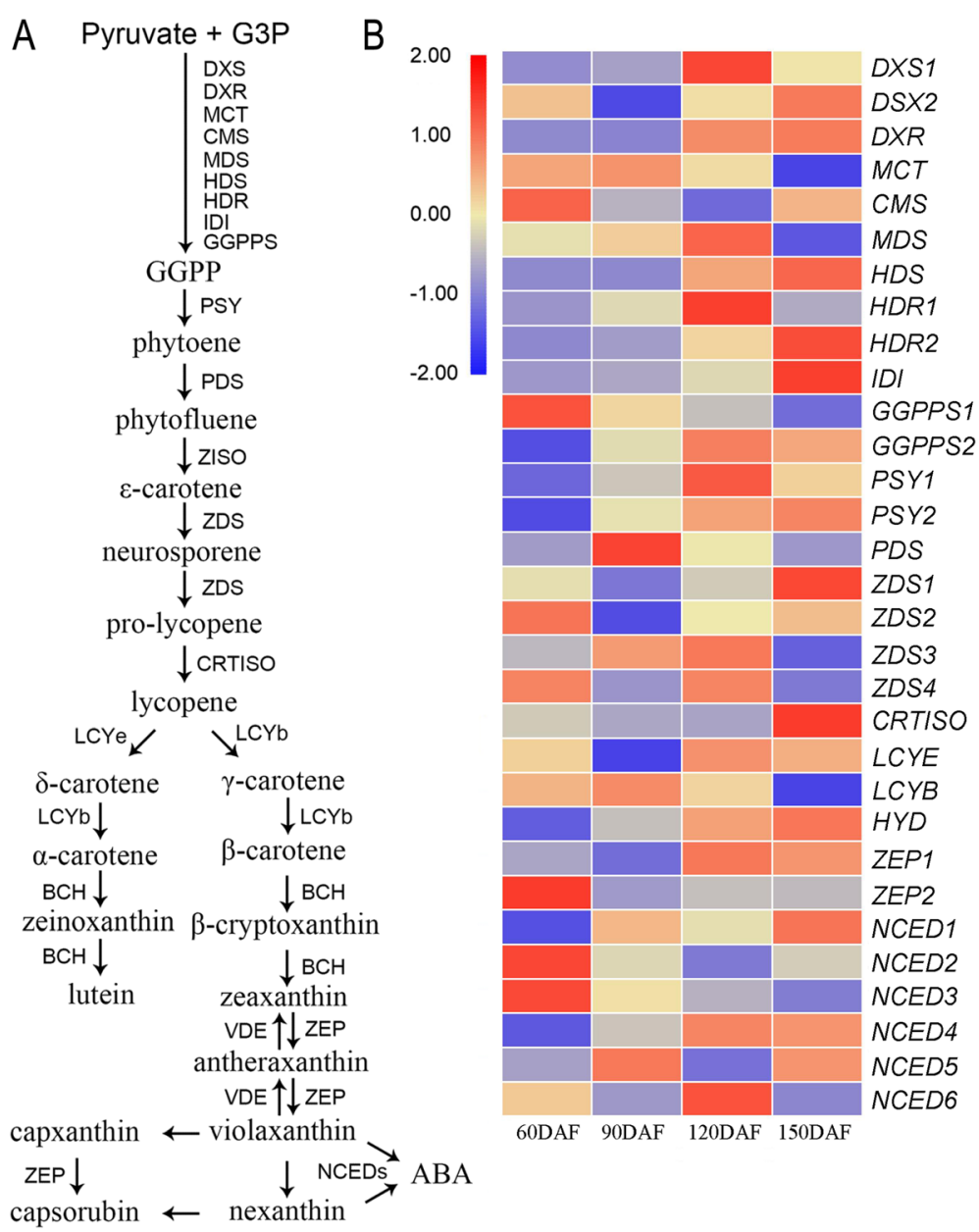

Fig. 4 Heatmap representation of the expression levels of carotenoid biosynthesis-related genes in CNO fruit based on transcriptome data. a, Carotenoid metabolism pathway in citrus. b. Expression level of carotenoid metabolism pathway genes in CNO fruit at four developmental stages

1: Table S1), maybe because the intermediate lycopene was over-accumulated, which may affect the biosynthesis of downstream carotenoids and other branch products. Similar to previous result, lycopene was found to be the most abundant carotenoid in $\mathrm{CNO}$ [6]. In the study, 90120 DAF was identified as the key stage for lycopene and carotenoid biosynthesis (Fig. 1a). However, 180 DAF was found to be the key stage for lycopene accumulation in a sweet orange, 'Hong Anliu' [20]. Thus, this study has for the first time revealed an early regulatory mechanism governing lycopene over-accumulation in $\mathrm{CNO}$ fruit.

\section{Important pathway genes contributing to carotenoids accumulation}

In MEP pathway, GGPP is the direct precursor for the production of the first carotenoid, the colorless phytoene, catalyzed by PSY $[8,18]$. However, DXS and DXR within MEP pathway play important roles in regulating the biosynthesis of carotenoid, abscisic acid, GAs and monoterpene [39]. To date, most of the carotenoid biosynthetic genes were cloned from citrus and other plants, such as GGPPS, PSY, PDS, ZDS, CRTISO, LCYB, $L C Y E, D H Y, Z E D$ and $C C D[8,12]$, among them, $P S Y$, $P D S, Z D S$ and $C R T I S O$ were upstream to lycopene biosynthesis, while $L C Y B, L C Y E, D H Y, Z E D$ and $C C D$ were downstream. It was reported that the expression level of PSY was related to carotenoids accumulation in tomato and citrus, and $P S Y$ played an important role in lycopene biosynthesis $[17,18]$. $L C Y B$ and $L C Y E$ contributed to the production of $\beta$-carotene and $\alpha$-carotene, respectively, using lycopene as a precursor [40]. The expression level of PSY in CNO was significantly higher than that in Washington navel orange fruit [41], and the higher expression levels of DXR and GGPPS were found in CNO fruit than in Washington navel orange fruit at the mature stage [29]. However, $P S Y$ and $L C Y B$ were found to be important for the accumulation of carotenoids in the 'Hong Anliu' sweet orange [17]. Previous studies showed that overexpression of $Z D S$ led to a more accumulation 


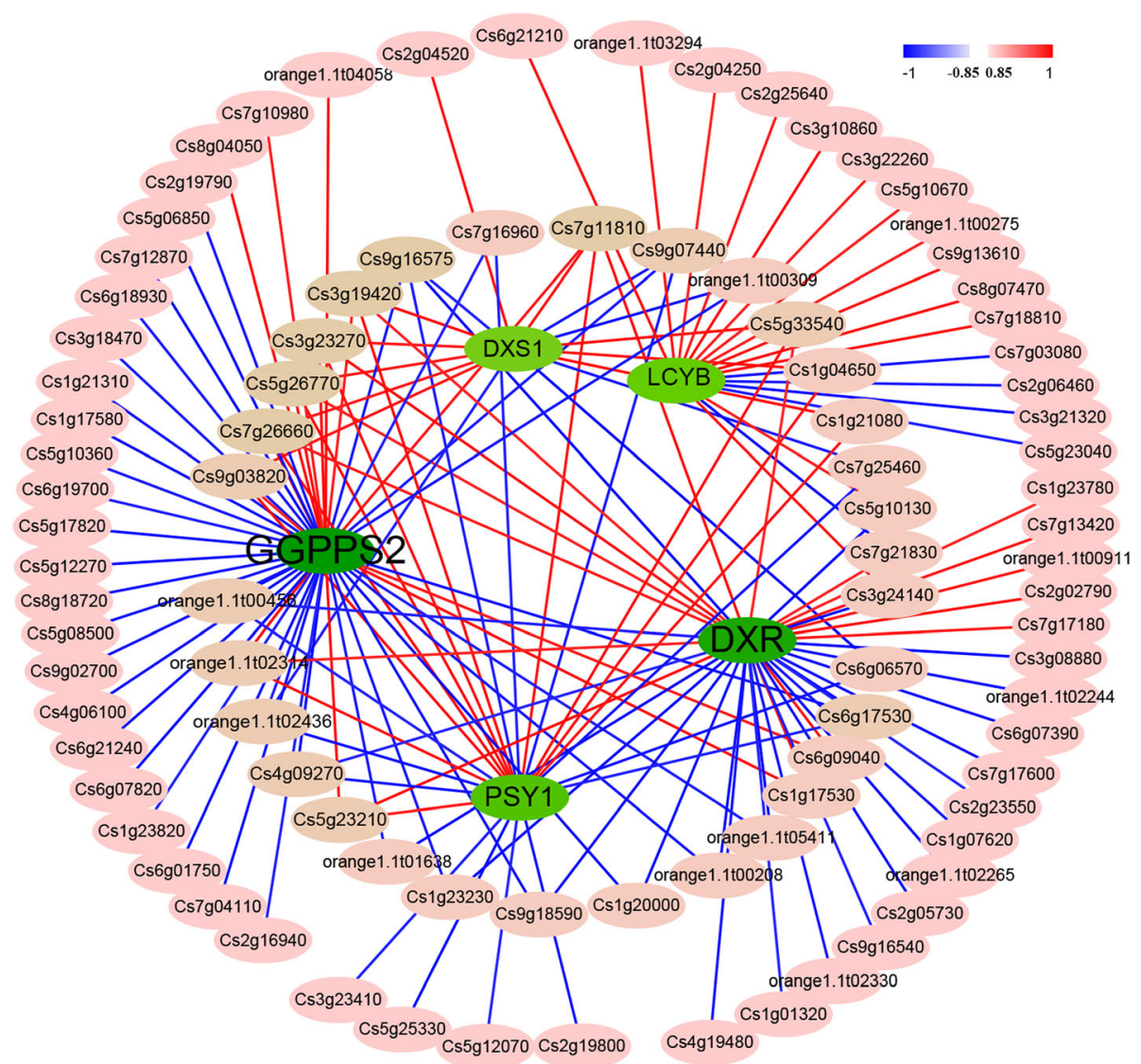

Fig. 5 The relationships between DXS1 (Cs1g20530), DXR (Cs5g05440), GGPPS2 (Cs8g02140), PSY1 (Cs6g15910) and LCYB (orange1.1 t00772) and its potential TFs based on transcriptome data

of upstream carotenoids in tomato [42], and in our study, ZDS3 expressed at 90 and 120 DAF stage, the key stage for lycopene and carotenoid biosynthesis, so we speculated that the ZDS3 might contribute to lycopene accumulation in $\mathrm{CNO}$.

In this study, the four genes, DXS1, DXR, GGPPS 2 and $P S Y 1$ were found to significantly change during fruit development, based on metabolomic data, KEGG and Mfuzz cluster, and also showed a high Pearson correlation values $(>0.80)$ (Additional file 3: Fig. S1; Fig.2; Table 1), indicating the important contribution of these genes to carotenoids accumulation in $\mathrm{CNO}$ fruit. The expression levels of these four genes were the highest at 120 DAF, coinciding with the highest increased rate of lycopene level from 90 to 120 DAF (Fig. 3). Although another significant increase of lycopene content happened from 120 to 150 DAF with a slightly lower rate compared to the previous period, the expression levels of the four genes at 150 DAF were lower than those at 90 and 120 DAF.

It is known that the accumulation of carotenoids is a net effect of several biological processes, including synthesis, transportation and degradation. The LCYE and
$L C Y B$ are key genes for the lycopene degradation [17, 43]. In our study, the $L C Y B$ expression maintained relative low level throughout fruit development (Fig. 3). Moreover, another lycopene accumulation-related gene $L C Y E$ was found to have a high Pearson correlation coefficient with lycopene content (Table 1). However, the contents of its direct products, $\alpha$-carotene and lutein, were retained at low levels. Therefor, in the study, our data indicated that the relative low enzymatic activity of $L C Y E$ together with the lower $L C Y B$ expression level before $150 \mathrm{DAF}$ was one of the most important reasons for the higher accumulation of lycopene.

Taken together, we speculated that high expression levels of DXS1, DXR, GGPPS2, PSY1 and relative low expression level of $L C Y B$ might play important role for lycopene accumulation in $\mathrm{CNO}$ fruit.

\section{Potential TFs may play important roles in lycopene accumulation}

TFs play important roles in plant biological regulatory networks. It was reported that some TFs regulated the biosynthesis of carotenoids. For example, CsMADS6 could bind $P S Y$ and $L C Y B$ promoters and directly 


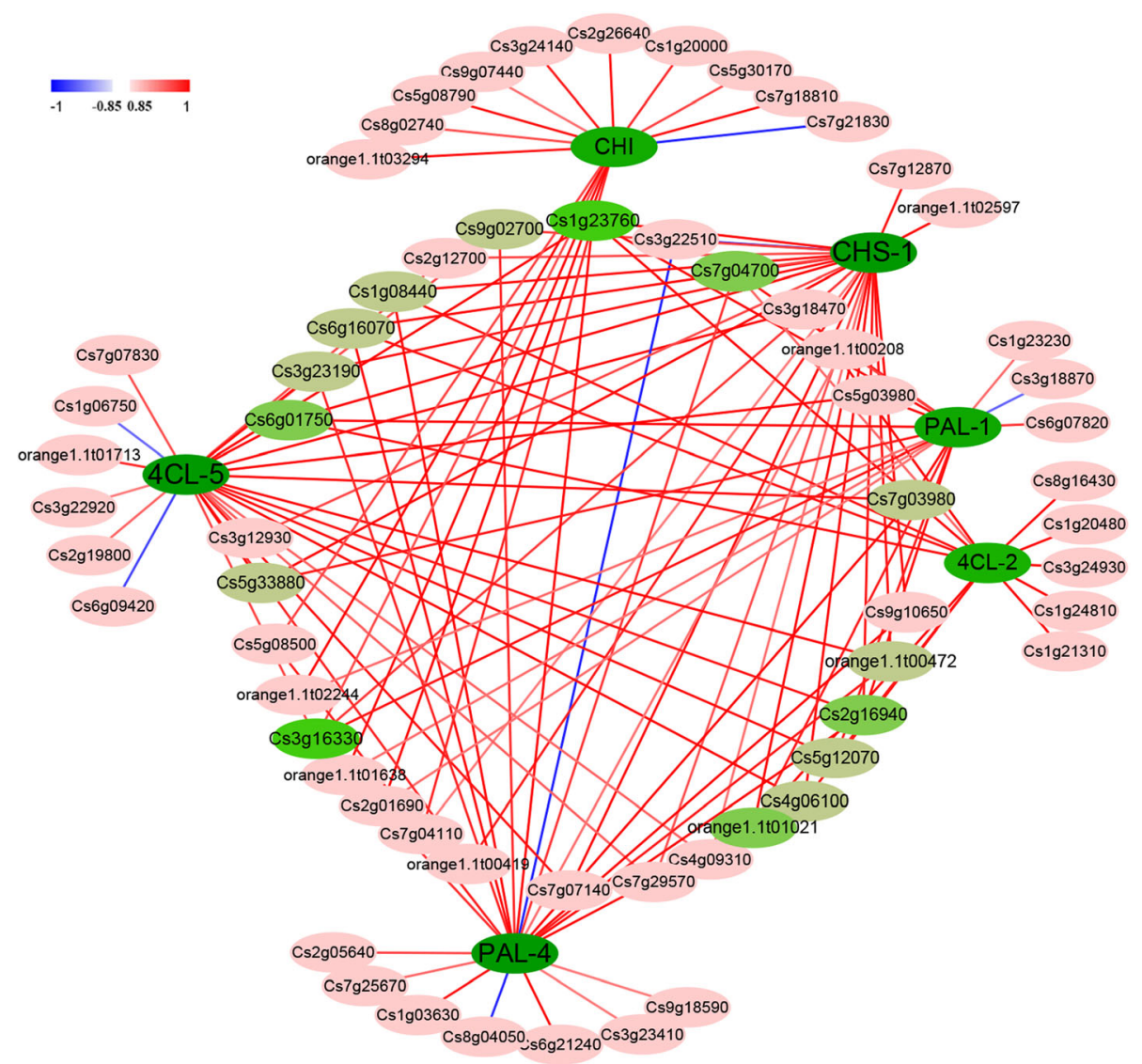

Fig. 6 The relationships between PAL-1 (Cs6g11940), PAL-4 (Cs8g16290), 4CL-2 (Cs5g24900), 4CL-5 (orange1.1 t04489), CHS-1 (Cs2g14720) and CHI (Cs7g28130) genes and its potential TFs based on transcriptome data

regulate their expression levels, and thus affect the accumulation of carotenoids in sweet orange fruit, 'Hong Anliu' [17], while PIFs, HY5, RAP2.2, RIN and SIMADS1 can directly bind the PSY promoter to alter the carotenoids profiles [25, 26, 44]. In this study, 16, 40, 48, 24 and 18 TFs showed high Pearson correlation coefficients $(|\mathrm{r}| \geq 0.85)$ with DXS1, DXR, GGPPS2, PSY1 and LCYB (Additional file 2: Table S7). The TFs were involved in AP2, GRAS, MADS, MYB and WRKY type transcription factors, were found to possibly bind DXS1, DXR, GGPP $S 2$ and PSY1 promoters (Additional file 2: Table S7). Eight and seven TFs may positively and negatively regulate at least three carotenoids biosynthetic pathway genes respectively, and thus may play important roles in the over-accumulation of lycopene in CNO. It was reported that over-expression of CUbHLH1 decreased lycopene content in tomato, while PpPLENA had the opposite regulation effect [22, 24]. And some TFs could affect plant photosynthesis and result in carotenoid accumulation rather than directly bind carotenoid biosynthetic genes, such as Gloden2-like in tomato [24]. Therefore, the TFs identified in the study might regulate other biological processes, such as hormone signal transduction and photosynthesis to affect the special carotenoids profile in the $\mathrm{CNO}$ fruit. However, as reported in 'Hong Anliu', the Pearson correlation coefficient was only - 0.762 between CsMADS6 (Cs7g16970) and PSY1, while other MADS TFs, Cs7g11810 and Cs1g21080 showed higher and positive Pearson correlation coefficients (>0.900) in CNO fruit, but it still need further confirmation by yeast one-hybrid $(\mathrm{Y} 1 \mathrm{H})$ and electrophoretic mobility shift assay (EMSA) study [17]. It would be of interest to find out if they are more important than CsMADS6 in lycopene accumulation.

\section{Pathway genes and TFs that may play important roles in flavonoids accumulation}

Flavonoids are important secondary metabolites in citrus fruit, and biosynthesized via phenylpropanoid biosynthetic pathway, with $P A L, C 4 H, 4 C L, C H S$ and $C H I$ genes involved in the pathway [32]. The contents of various flavonoids were stable or decreased during fruit development, and the expression levels of $P A L, C 4 H, 4 C L$, CHS-1 and CHI were especially-expressed at $60 \mathrm{DAF}$ and significantly decreased at 90, 120 and 210 DAF in 'Fenghuang' pomelo, 'KaoPan' pomelo, 'Huanong red' 
pomelo and 'HB' pomelo [32]. Similarly in this study, the expression levels of PAL-1, PAL-3, 4CL-2, 4CL-5, CHS-1 and $\mathrm{CHI}$ genes were found to decrease during fruit development (Additional file 4: Fig. S2), indicating the important roles of these genes for flavonoids accumulation at early stages.

Many TFs were reported to regulate phenylpropanoid biosynthetic pathway genes, and then affect the contents of anthocyanin, lignin and flavonoids. For example, MYBF1 was found to bind CHS promoter and then regulate the synthesis of flavonol in citrus and grapevine $[34,37]$, while many other MYB TFs were reported to bind PAL, $4 C L$ and $C H S$ promoters and then regulate anthocyanin and lignin biosynthesis [44]. In the study, 64 potential TFs with high Pearson correlation coefficients were predicted based on PAL-1, PAL-3, 4CL-2, $4 C L-5, C H S-1$ and CHI promoters' analysis (Fig. 6). Some of them could potentially bind promoters of multiple genes, and thus may be most important TFs regulating flavonoids accumulation in $\mathrm{CNO}$ fruit.

\section{Potential TFs that may co-regulate carotenoids and flavonoids accumulation}

In the study, in $\mathrm{CNO}$ fruit, the content of carotenoids and expression levels of key genes involved in carotenoids synthesis tended to increase, while the level of flavonoids and expression levels of the key genes tended to decrease (Fig. 1; Fig. 3). Further analysis revealed that both pathways' key genes might be co-regulated by 24 potential TFs directly (Additional file 6: Fig. S4). For instance, Cs1g21310 might positively and negatively regulate flavonoids and carotenoids accumulation. Considering the potential TFs with direct functions in carotenoids and flavonoids biosynthesis, 24 TFs might serve as important TFs for the accumulation of carotenoids and flavonoids in $\mathrm{CNO}$ fruits to form a regulatory network (Additional file 6: Fig. S4). These TFs may form an integrative fruit quality regulatory network with various metabolic pathways in fruits.

\section{Conclusions}

Carotenoids and flavonoids are important metabolites in citrus fruit, and lycopene was found to be the major compound contributing to the red flesh in $\mathrm{CNO}$, while narirutin as the dominant flavonoid at the early stage. Carotenoids and flavonoids profiling of $\mathrm{CNO}$ juice sacs at six developmental stages identified $90-120$ DAF as the key stage for the over-accumulation of lycopene. Transcriptomic analysis indicated that DXS1, DXR, GGPPS2, PSY1 and $L C Y B$ genes were the most important genes for carotenoids accumulation, while $P A L-1$, PAL-4, 4CL-2, 4CL-5, CHS-1 and CHI for flavonoids biosynthesis. Both sets of 15 candidate TFs were found to potentially bind the promoters of at least three carotenoids/flavonoids key pathway genes to regulate their expression levels, and thus help generate the special carotenoids and flavonoids profiles in CNO fruits. In addition, a total of 24 TFs were postulated as coregulators in both pathways directly.

\section{Methods \\ Materials}

The fruit of CNO (Citrus sinensis Osbeck) were collected from the National Citrus Breeding Center (Wuhan, Hubei Province, China), the CNO cultivar was identified by the germplasm nursery manager of Zongzhou Xie. The fruit were harvested from the adult and healthy trees under normal management at six fruit development stages of $60,90,120,150,180$ and 210 DAF. Three biological replicates per stage were analyzed, with each replicate consisting of six healthy and similar sizes fruit from three different plants. The juice sacs were immediately separated with a scalpel, and then placed in liquid nitrogen and stored at $-80{ }^{\circ} \mathrm{C}$ until analysis.

\section{Extraction and identification of carotenoids}

Carotenoids were extracted from $2 \mathrm{~g}$ juice sacs and then measured according to Liu et al. [20]. Briefly, $2 \mathrm{~g}$ juice sacs were grounded into power in liquid nitrogen for the extraction. Carotenoids profiling were conducted by high-performance liquid chromatography (HPLC, Waters 1525 reverse-phase HPLC with a 2996 photodiode array detector) with a C30 carotenoid column $(250 \mathrm{~mm}$ $\times 4.6 \mathrm{~mm}, \mathrm{YMC}$, Wilmington, NC, USA). The carotenoids were identified basing on the retention times and the absorption spectra with authentic standards according to our previous studies [20].

\section{Determination of flavonoids}

Flavonoids were extracted from $1 \mathrm{~g}$ juice sacs for each sample using the method according to Chen et al. [9] with minor modification. The samples were grounded into power in liquid nitrogen, $1 \mathrm{~g}$ power were then used to flavonpids extraction in $2 \mathrm{~mL} 80 \%$ aqueous methanol for $60 \mathrm{~min}$ at $40^{\circ} \mathrm{C}$ in ultrasonic bath model FS60 (Fisher Scientific, Pittsburgh, PA). The extraction were centrifuged at $10,000 \times \mathrm{g}$ for $10 \mathrm{~min}$, then the supernatant was filtered through a $0.22 \mu \mathrm{m}$ micropore and analyzed using HPLC. Flavonoids were analyzed using the HPLC system with a C18 Hypersil GOLD column $(250 \mathrm{~mm} \times 4.6 \mathrm{~mm}$ $\times 5 \mu \mathrm{m}$, Thermo Scientific). The parameters for HPLC of flavonoids were according Chen et al. [9].

\section{RNA extraction, Transcriptomic sequencing and analysis}

The CNO juice sacs at 60, 90, 120 and 150 DAF stages were used to total RNA extraction as described by Liu et al. [45], $2 \mu \mathrm{g}$ total RNA for each sample was used for cDNA library construction according to the protocol 
(TruSeq RNA Sample Preparation v2 Guide, Illumina, United States). The mRNA was purified using the Illumina TruSeq RNA Sample Prep Kit v2, and then assessed using the Agilent Technologies 2100 Bioanalyzer (Agilent, United States). The first- and second-strand complementary DNA was synthesized, double-strand cDNA was purified, the adapters were added, and then the mRNA was cleaved into short fragments of approximately $270 \mathrm{bp}$. The constructed libraries were sequenced on an Illumina HiSeq 4000 platform in a paired-end (2 $\times 100 \mathrm{bp}$ ) mode by Millennium Co. (Seoul, Korea). The RNA-seq raw-data were uploaded onto NCBI (SRA) under the accession number PRJNA636131.

The genome and annotation files of Valencia orange (C. sinensis) were downloaded from the Orange Genome Annotation Project (http://citrus.hzau.edu.cn/orange/ download/ index.php). A reference index by STAR (version 2.7) was built. Raw data were filtered using Trimmomatic (0.36) under default parameters, then the clean data were mapped to reference genome using STAR, and the counts calculated using Htseq-count based on mapping results. The edge $\mathrm{R}$ was used to determine the differentially expressed genes, and then the DEGs were used for function annotation based on the Mercator sequence annotation software (https://www.plabipd.de/ portal/web/guest/home1). The DEGs were subjected to KEGG enrichment analysis and GO annotation using KOBAS 3.0 (http://kobas.cbi.pku.edu.cn/) and TBtools [46], respectively. The expressed genes were clustered into nine groups using the Mfuzz package in $\mathrm{R}$.

\section{Prediction of potential binding TF}

The promoter sequences of DXS1 (Cs1g20530), DXR (Cs5g05440), GGPPS2 (Cs8g02140), PSY1 (Cs6g15910), LCYB (orange1.1 t00772), PAL-1 (Cs6g11940), PAL-4 (Cs8g16290), 4CL-2 (Cs5g24900), 4CL-5 (orange1.1 t04489), CHS-1 (Cs2g14720) and CHI (Cs7g28130) were used to predict the binding TFs using the Regulation Prediction function in PlantRegMap (http://plantregmap.cbi.pku.edu.cn/index. php). The $C$. sinensis database was used as a library, and $P$-value was lower than $1 \mathrm{e}^{-5}$. The TFs were converted to the similar genes in Valencia sweet orange genome using BLAST function. All of the TFs in MADS, AP2 and GRAS (based on Mercator v.3.6 annotation results) were selected for further analysis. Pearson correlation coefficients between the above pathway genes with potential binding TFs were calculated, based on transcriptome data by $\mathrm{R}$ or function with 'Pearson' method. The Pearson correlation coefficient value was filtered by higher than 0.850 or lower than -0.850 , and the results were shown using Cytoscapev3.7.2.

\section{Data analysis}

The contents of carotenoids and flavonoids were calculated using the standard curves. The R packages (nortest, stats and pgirmess) were used for analysis of variance with ANOVA $(P<0.05)$. Bar chart and dot charts were made based on DEGs and KEGG analysis results using $R$ package, ggplot2. A heatmap of carotenoids biosynthesis-related genes was constructed based on transcriptome data using TBtools [46].

\section{Supplementary Information}

The online version contains supplementary material available at https://doi. org/10.1186/s12870-020-02808-3.

Additional file 1: Table S1. Carotenoids identified in CNO at six developmental stages ( $\mu \mathrm{g} / \mathrm{g}, \mathrm{FW}$ ). U: undetectable. Table S2. Flavonoids identified in CNO at six developmental stages (ng/g, FW). U:

undetectable. Table S3. Basic statistics of RNA-Seq clean-data mapped to the reference genome. Table S4. The expression levels of genes in CNO fruit (FPKM). Table S5. The expressed genes were clustered into nine groups by Mfuzz.

Additional file 2: Table S6. TFs were predicted in DXS1, DXR, GGPPS2, PSY1 and LCYB. Table S7. The TFs that showed high Pearson correlation coefficients with carotenoids synthesis-related genes. PCC ${ }^{\mathrm{a}}$ : Pearson correlation coefficient. Table S8. TFs predicted in PAL-1, PAL-4, 4CL-2, 4CL-5, CHS- 1 and $\mathrm{CHI}$ genes. Table S9. The TFs that showed high Pearson correlation coefficients with flavonoids synthesis-related genes. PCC $C^{\text {: }}$ Pearson correlation coefficient. Table S10. TFs predicted in 30 most important TFs that regulate at least three carotenoid or flavonoids genes.

Additional file 3: Figure S1. Differentially expressed genes in juice sacs of $\mathrm{CNO}$ fruit at different development stages. A, Numbers of differentially expressed genes at different developmental stages. B, KEGG analysis result of the differentially expressed genes in juice sacs between 90 DAF and 120 DAF.

Additional file 4: Figure S2. A heatmap showing the expression levels of flavonoids biosynthesis-related genes in CNO fruit based on transcriptome data.

Additional file 5: Figure S3. KEGG analysis result of the differentially expressed genes in juice sacs between 60 DAF and 90 DAF.

Additional file 6: Figure S4. TFs that regulated the carotenoids and flavonoids biosynthesis genes.

\section{Abbreviations}

DXS: 1-deoxy-D-xylulose-5-phosphate synthase; DXR: Deoxyxylulose 5phosphate reductoisomerase; GGPPS: Geranylgeranyl diphosphate synthase; PSY: Phytoene synthase; LCYB: Lycopene $\beta$-cyclase; LCYE: Lycopene epsiloncyclase; PAL: Phenylalanine ammonia-lyase; 4CL: 4-Coumarate-CoA ligase; CHS: Chalcone synthase; CHI: Chalcone isomerase; CNO: Cara cara navel orange; TFs: Transcription factors; DAF: Days after florescence

\section{Acknowledgements}

We'll appreciate Mr. Zongzhou Xie from Huazhong Agricultural University for his help in sample collection.

\section{Authors' contributions}

HZ (Haipeng Zhang), JX and HYZ (Hongyan Zhang) designed the experiments; $\mathrm{HZ}$ performed most of the experiments; JC, ZP and XL performed part of the experiments; MS and HW analyzed the carotenoids data; $\mathrm{HZ}$ and $\mathrm{YJ}$ extracted the RNA; $\mathrm{HZ}$ and $\mathrm{HYZ}$ wrote the manuscript. JX, $Y C$ and HYZ reviewed and edited the manuscript. All authors agree with the manuscript contents and with its submission. All authors read and approved the final manuscript. 


\section{Funding}

This research was funded by the National Key Research and Development Program (2018YFD1000200), the National Natural Science Foundation of China (NSFC 31672102) and The Fundamental Research Funds for the Central Universities (2662018PY072). The funders had no role in the design of the study; in the collection, analyses, or in the preparation of data; in the writing of the manuscript, or in the decision to publish the results.

\section{Availability of data and materials}

All data generated or analyzed during this study were included in this published article and the additional files. The RNA-seq data are available from the NCBI under the accession number PRJNA636131.

\section{Ethics approval and consent to participate}

The authors were complied by the national laws and the ethical approval in this study.

\section{Consent for publication}

Not applicable.

\section{Competing interests}

The authors declare that they have no competing interests.

Received: 28 July 2020 Accepted: 20 December 2020

Published online: 07 January 2021

\section{References}

1. Zhang $H$, Wen $H$, Chen J, Peng Z, Shi M, Chen M, Yuan Z, Liu Y, Zhang H, $\mathrm{Xu} \mathrm{J}$. Volatile compounds in fruit peels as novel biomarkers for the identification of four Citrus species. Molecules. 2019;24:4550.

2. Zhang H, Xie Y, Liu C, Chen S, Hu S, Xie Z, Deng X, Xu J. Comprehensive comparative analysis of volatile compounds in citrus fruits of different species. Food Chem. 2017;230:316-26.

3. Li W, Liu C, He M, Li J, Cai Y, Ma Y, Xu J. Largely different contents of terpenoids in beef red-flesh tangerine and its wild type. BMC Plant Biol. 2017; 17:36.

4. Wang L, He F, Huang Y, He J, Yang S, Zeng J, Deng C, Jiang X, Fang Y, Wen S. Genome of wild mandarin and domestication history of mandarin. Mol Plant. 2018;11:1024-37.

5. Xu J, Ma L, Jiang D, Zhu S, Yan F, Xie Y, Xie Z, Guo W, Deng X. Content evaluation of 4 furanocoumarin monomers in various citrus germplasms. Food Chem. 2015;187:75-81.

6. Xu J, Deng X. Identification of Main Pigments in Red Flesh Navel Orange (Citrus sinensis L.) and Evaluation of Their Concentration Changes during Fruit Development and Storage. Acta Horti Sin. 2002;29:203-8.

7. Fiedor J, Burda K. Potential role of carotenoids as antioxidants in human health and disease. Nutrients. 2014;6:466-88.

8. Hirschberg J. Carotenoid biosynthesis in flowering plants. Curr Opin Plant Biol. 2001:4:210-8.

9. Chen J, Zhang $H$, Pang $Y$, Cheng $Y$, Deng $X, X u J$. Comparative study of flavonoid production in lycopene-accumulated and blonde-flesh sweet oranges (Citrus sinensis) during fruit development. Food Chem. 2015;184 238-46.

10. Huang D, Wang $X$, Tang $Z$, Yuan $Y, X u$ Y, He J, Jiang X, Peng S, Li L, Butelli E. Subfunctionalization of the Ruby2-Ruby 1 gene cluster during the domestication of citrus. Nat plants. 2018;4:930-41.

11. Kato M, Ikoma Y, Matsumoto H, Sugiura M, Hyodo H, Yano M. Accumulation of carotenoids and expression of carotenoid biosynthetic genes during maturation in citrus fruit. Plant Physiol. 2004;134:824-37.

12. Zheng X, Zhu K, Sun Q, Zhang W, Wang X, Cao H, Tan M, Xie Z, Zeng Y, Ye J. Natural variation in CCD4 promoter underpins species-specific evolution of red coloration in citrus peel. Mol Plant. 2019;12:1294-307.

13. Kim MJ, Kim H. Anticancer effect of lycopene in gastric carcinogenesis. J Cancer Prev. 2015;20:92.

14. Lois LM, Rodríguez-Concepción M, Gallego F, Campos N, Boronat A. Carotenoid biosynthesis during tomato fruit development: regulatory role of 1-deoxy-D-xylulose 5-phosphate synthase. Plant J. 2000;22:503-13.

15. Nieuwenhuizen NJ, Chen X, Wang M, Matich A, Perez RL, Allan AC, Green SA, Atkinson RG. Natural variation in monoterpene synthesis in kiwifruit: transcriptional regulation of terpene synthases by NAC and ETHYLENE-INSE NSITIVE3-like transcription factors. Plant Physiol. 2015;167:1243-58.
16. Mahmoud SS, Croteau RB. Metabolic engineering of essential oil yield and composition in mint by altering expression of deoxyxylulose phosphate reductoisomerase and menthofuran synthase. P Natl A Sci. 2001;98:8915-20.

17. Lu S, Zhang Y, Zhu K, Yang W, Ye J, Chai L, Xu Q, Deng X. The citrus transcription factor CsMADS6 modulates carotenoid metabolism by directly regulating carotenogenic genes. Plant Physiol. 2018;176:2657-76.

18. Giuliano G, Bartley GE, Scolnik PA. Regulation of carotenoid biosynthesis during tomato development. Plant Cell. 1993;5:379-87.

19. Luo Z, Zhang J, Li J, Yang C, Wang T, Ouyang B, Li H, Giovannoni J, Ye Z. A STAY-GREEN protein SISGR1 regulates lycopene and $\beta$-carotene accumulation by interacting directly with SIPSY1 during ripening processes in tomato. New Phytol. 2013;198:442-52.

20. Liu Q, Xu J, Liu Y, Zhao X, Deng X, Guo L, Gu J. A novel bud mutation that confers abnormal patterns of lycopene accumulation in sweet orange fruit (Citrus sinensis L. Osbeck). J Exp Bot. 2007;58:4161-71.

21. Zeng Y, Pan Z, Ding Y, Zhu A, Cao H, Xu Q, Deng X. A proteomic analysis of the chromoplasts isolated from sweet orange fruits [Citrus sinensis $(\mathrm{L}$.) Osbeck]. J Exp Bot. 2011;62:5297-309.

22. Itkin M, Seybold H, Breitel D, Rogachev I, Meir S, Aharoni A. TOMATO AGAMOUS-LIKE 1 is a component of the fruit ripening regulatory network. Plant J. 2009:60:1081-95.

23. Lee JM, Joung JG, McQuinn R, Chung MY, Fei Z, Tieman D, Klee H, Giovannoni J. Combined transcriptome, genetic diversity and metabolite profiling in tomato fruit reveals that the ethylene response factor SIERF6 plays an important role in ripening and carotenoid accumulation. Plant J. 2012;70:191-204.

24. Vrebalov J, Pan IL, Arroyo AJM, McQuinn R, Chung M, Poole M, Rose J, Seymour G, Grandillo S, Giovannoni J. Fleshy fruit expansion and ripening are regulated by the tomato SHATTERPROOF gene TAGL1. Plant Cell. 2009; 21:3041-62.

25. Dong T, Hu Z, Deng L, Wang Y, Zhu M, Zhang J, Chen G. A tomato MADSbox transcription factor, SIMADS1, acts as a negative regulator of fruit ripening. Plant Physiol. 2013;163:1026-36.

26. Toledo-Ortiz G, Huq E, Rodríguez-Concepción M. Direct regulation of phytoene synthase gene expression and carotenoid biosynthesis by phytochrome-interacting factors. P Natl A Sci. 2010;107:11626-31.

27. Toledo-Ortiz G, Johansson H, Lee KP, Bou-Torrent J, Stewart K, Steel G, Rodríguez-Concepción M, Halliday KJ. The HY5-PIF regulatory module coordinates light and temperature control of photosynthetic gene transcription. PLoS Genet. 2014;10:e1004416.

28. Zhu F, Luo T, Liu C, Wang Y, Yang H, Yang W, Zheng L, Xiao X, Zhang M, Xu R. An R2R3-MYB transcription factor represses the transformation of a-and $\beta$-branch carotenoids by negatively regulating expression of $\mathrm{CrBCH} 2$ and CrNCED5 in flavedo of Citrus reticulate. New Phytol. 2017;216:178-92.

29. Pan Z, Zeng Y, An J, Ye J, Xu Q, Deng X. An integrative analysis of transcriptome and proteome provides new insights into carotenoid biosynthesis and regulation in sweet orange fruits. J Proteome. 2012;75: 2670-84.

30. Tripoli E, Guardia ML, Giammanco S, Majo DD, Giammanco M. Citrus flavonoids: molecular structure, biological activity and nutritional properties: a review. Food Chem. 2007:104:466-79.

31. Nakajima A, Ohizumi Y. Potential benefits of Nobiletin, a Citrus flavonoid, against Alzheimer's disease and Parkinson's disease. Int J Mol Sci. 2019;20:3380

32. Chen J, Yuan Z, Zhang H, Li W, Shi M, Peng Z, Li M, Tian J, Deng X, Cheng Y. Cit1,2RhaT and two novel CitdG/cTs participate in flavor-related flavonoid metabolism during citrus fruit development. J Exp Bot. 2019;70:2759-71.

33. Jia N, Liu J, Tan P, Sun Y, Lv Y, Liu J, Sun J, Huang Y, Lu J, Jin N. Citrus sinensis MYB transcription factor CsMYB85 induce fruit juice sac lignification through interaction with other CsMYB transcription factors. Front Plant Sci. 2019:10:213.

34. Liu C, Long J, Zhu K, Liu L, Yang W, Zhang H, Li L, Xu Q, Deng X. Characterization of a Citrus R2R3-MYB transcription factor that regulates the Flavonol and Hydroxycinnamic acid biosynthesis. Sci Rep. 2016;6:25352.

35. Butelli E, Licciardello C, Zhang Y, Liu J, Mackay S, Bailey P, ReforgiatoRecupero G, Martin C. Retrotransposons control fruit-specific, colddependent accumulation of anthocyanins in blood oranges. Plant Cell. 2012;24:1242-55.

36. Jia N, Liu J, Sun Y, Tan P, Cao H, Xie Y, Wen B, Gu T, Liu J, Li M. Citrus sinensis MYB transcription factors CsMYB330 and CsMYB308 regulate fruit juice sac lignification through fine-tuning expression of the Cs4CL1 gene. Plant Sci. 2018;277:334-43. 
37. Comeskey DJ, Montefiori M, Edwards PJ, MCGhie TK. Isolation and structural identification of the anthocyanin components of red kiwifruit. J Agri Food Chem. 2009;57:2035-9.

38. Bartley GE, Scolnik PA. Plant carotenoids: pigments for photoprotection, visual attraction, and human health. Plant Cell. 1995;7:1027-38.

39. Matthews $P$, Wurtzel DET. Metabolic engineering of carotenoid accumulation in Escherichia coli by modulation of the isoprenoid precursor pool with expression of deoxyxylulose phosphate synthase. Appl Microbiol Biot. 2000;53:396-400.

40. Harjes CE, Rocheford TR, Bai L, Brutnell TP, Kandianis CB, Sowinski SG, Stapleton AE, Vallabhaneni R, Williams M, Wurtzel ET. Natural genetic variation in lycopene epsilon cyclase tapped for maize biofortification. Science. 2008;319:330-3.

41. Tao N, Hu Z, Liu Q, Xu J, Cheng Y, Guo L, Guo W, Deng X. Expression of phytoene synthase gene (Psy) is enhanced during fruit ripening of Cara Cara navel orange (Citrus sinensis Osbeck). Plant Cell Rep. 2007;26:837-43.

42. Mcquinn RP, Gapper NE, Gray AG, Zhong S, Tphge T, Fei ZJ, Fernie AR, Giovannoni JJ. Manipulation of ZDS in tomato exposes carotenoid- and ABA-specific effects on fruit development and ripening. Plant Biotechnol J. 2020;18:2210-24.

43. Ronen G, Cohen M, Zamir D, Hirschberg J. Regulation of carotenoid biosynthesis during tomato fruit development: expression of the gene for lycopene epsilon-cyclase is down-regulated during ripening and is elevated in the mutant Delta. Plant J. 1999;17:341-51.

44. Ma D, Constabel CP. MYB repressors as regulators of Phenylpropanoid metabolism in plants. Trends Plant Sci. 2019:24:275-89.

45. Liu YZ. Efficient isolation of RNA from fruit Peel and pulp of ripening navel Orange (Citrus sinensis Osbeck). J Huazhong Agricult Univ. 2006;25:300-4.

46. Chen C, Xia R, Chen H, He Y. TBtools: an integrative toolkit developed for interactive analyses of big biological data. Mol Plant. 2020;13:1194-202.

\section{Publisher's Note}

Springer Nature remains neutral with regard to jurisdictional claims in published maps and institutional affiliations.

Ready to submit your research? Choose BMC and benefit from:

- fast, convenient online submission

- thorough peer review by experienced researchers in your field

- rapid publication on acceptance

- support for research data, including large and complex data types

- gold Open Access which fosters wider collaboration and increased citations

- maximum visibility for your research: over $100 \mathrm{M}$ website views per year

At $\mathrm{BMC}$, research is always in progress.

Learn more biomedcentral.com/submissions 